\title{
Alfabetización tecnológica en estudiantes de nivel superior
}

\section{Technological literacy in higher level students}

PREZA-MEDINA, Sergio Roberto*†, HERNÁNDEZ-CHACÓN, Sandra, CEBOLLÓN-MEZA, Ángela y SAAVEDRA-RIVEROS, Érika

Universidad Tecnológica de Cancún. Carretera Cancún-Aeropuerto, Km. 11.5 S.M. 299, Mz. 5, Lt 1, 77565 Cancùn, Q.R.

ID $1^{\text {er }}$ Autor: Sergio Roberto, Preza-Medina / ORC ID: 0000-0001-5010-0585, CVU CONACYT ID: 505691

ID $1^{\text {er }}$ Coautor: Sandra, Hernández-Chacón / ORC ID: 0000-0002-0845-8791, CVU CONACYT ID: 742521

ID $2^{\text {do }}$ Coautor: Ángela, Cebollón-Meza / ORC ID: 0000-0002-2588-061X, CVU CONACYT ID: 747569

ID $3^{\text {er }}$ Coautor: Érika, Saavedra-Riveros / ORC ID: 0000-0002-3094-063X, CVU CONACYT ID: 900083

DOI: $10.35429 / J E T .2019 .10 .3 .27 .33$

Recibido: 02 de Noviembre, 2019; Aceptado 28 de Diciembre, 2019

\section{Resumen}

El objetivo general de este proyecto fue identificar el nivel de alfabetización tecnológica que tienen los estudiantes que cursan el tercer cuatrimestre del programa educativo de Tecnologías de la Información y Comunicación En cuanto a la metodología, se optó por la aplicación del instrumento Cuestionario para el Estudio de la Competencia Digital del Alumnado de Educación Superior (CDAES) Gutiérrez, J.; Cabero, J.\& Estrada. L. (2017). De igual manera, la muestra objeto de estudio, fue seleccionada de entre los estudiantes universitarios, matriculados en algún grupo del programa educativo de TSU en TIC. Se observó que el nivel de alfabetización tecnológica en los estudiantes del tercer cuatrimestre en el programa educativo de Tecnologías de la Información y Comunicación en un $23.8 \%$, el resultado fue "Bien" y el $76.2 \%$ fue "Excelente".

Alfabetización Tecnologica, CDAES competencias

\begin{abstract}
The integral formation of the university students is of great The general objective of this project was to identify the level of technological literacy students of IT and Systems Engineering. Regarding the methodology, it was applied an instrument called "Questionnaire for the study of Digital Competence of University Students CDAES) Gutiérrez, J.; Cabero, J.\& Estrada. L. (2017). Also, the analysis simple was chosen among university students enrolled in IT programs. It was observed the level of Technological literacy in students of third quarter in the IT and Systems Engineering program in a $23.8 \%$ the result was well and $76.2 \%$ was excellent.
\end{abstract}

Citación: PREZA-MEDINA, Sergio Roberto, HERNÁNDEZ-CHACÓN, Sandra, CEBOLLÓN-MEZA, Ángela y SAAVEDRA-RIVEROS, Érika. Alfabetización tecnológica en estudiantes de nivel superior. Revista Teoría Educativa. 2019. 3-10: 27-33.

\footnotetext{
* Correspondencia del Autor (Correo electrónico: spreza@utcancun.edu.mx)

$\dagger$ Investigador contribuyendo como primer autor.
} 


\section{Introducción}

El quehacer educativo exige a las nuevas generaciones de estudiantes tener un amplio dominio de las herramientas tecnológicas y de comunicación. Así, la nueva sociedad de la información demanda que las instituciones educativas fortalezcan el compromiso de brindar a los educandos dicha alfabetización para lograr un desempeño óptimo en el mundo profesional.

De esta forma, es necesario el establecimiento de dominios de información, uso adecuado de la tecnología y habilidades de búsqueda, por citar algunas de las actividades informáticas, que deben ser consideradas en los programas de estudio. En lo que refiere a la parte del docente, éste muestra un interés genuino en que su enseñanza contemple las diferentes herramientas tecnológicas a su favor, además de promover en el estudiante la autogestión, administración del tiempo y adopción de estrategias de estudio para el aprendizaje en línea. Bajo esta argumentación, resulta imperativo determinar el nivel de alfabetización tecnológica de los estudiantes.

\section{Alfabetización tecnológica en estudiantes de nivel superior}

\section{Problema}

En la universidad donde se realiza el estudio no se cuenta con información precisa sobre qué nivel de alfabetización tecnológica tienen los estudiantes que cursan el tercer cuatrimestre del programa educativo de Tecnologías de la Información y Comunicación.

\section{Preguntas de investigación}

¿Los estudiantes entienden y usan sistemas informáticos de comunicación e información?

¿Los estudiantes seleccionan y usan aplicaciones efectiva y productivamente?

¿Los estudiantes investigan y resuelven problemas en los sistemas y las aplicaciones?

¿Los estudiantes transfieren el conocimiento existente al aprendizaje de nuevas tecnologías de Información y Comunicación?

\section{Justificación}

El mundo de educación superior conlleva el dominio tecnológico y de información. Por ende, la alfabetización tecnológica es un factor medular en el desarrollo profesional de los estudiantes. Es la suma de todas estas habilidades, conocimientos y actitudes, en aspectos tecnológicos, informacionales, multimedia y comunicativos, dando lugar a una compleja alfabetización múltiple. Asimismo, y tal y como afirma Peña (2006), es una completa alfabetización funcional, ya que comprende la utilización de las herramientas de forma productiva, mucho más allá que un uso estrictamente operacional.

Gisbert (2011) destaca, que si estamos conscientes que ésta es una competencia clave que el estudiante debe desarrollar a lo largo de su proceso formativo en la universidad, debemos diseñar estrategias adecuadas que nos permitan tener la certeza de que los estudiantes la han adquirido. Para ello, deberíamos primero determinar qué nivel de alfabetización tecnológica tienen éstos cuando llegan a la universidad y poder garantizar, a través de su formación, que la tienen adquirida al finalizar sus estudios universitarios. Considerando que los grados universitarios deben capacitar para la incorporación al mercado laboral y profesional, $\mathrm{y}$ asumiendo que el entorno laboral es un entorno altamente digitalizado debemos de responsabilizarnos de garantizar esta competencia en todos los estudiantes.

La investigación planteada contribuirá a generar un modelo para conocer el nivel de alfabetización tecnológica que tienen los estudiantes del tercer cuatrimestre en el programa educativo de Tecnologías de la Información y Comunicación (TIC) Asimismo, los resultados del estudio ayudarán a mejorar los procesos de aprendizaje de éstos pasa por diseñar procesos formativos con unos objetivos educativos muy bien definidos, con una adecuada planificación de actividades y con el diseño de un proceso de evaluación de los aprendizajes coherente con la opción metodológica por la que hayamos optado. 


\section{Objetivo}

Identificar el nivel de alfabetización tecnológica en los estudiantes del tercer cuatrimestre en el programa educativo de Tecnologías de la Información y Comunicación.

\section{Objetivos específicos:}

1. Identificar las competencias que tienen los estudiantes para saber utilizar sistemas informáticos de comunicación e información.

2. Conocer el domino que tienen los estudiantes en el uso de herramientas ofimáticas para el tratamiento de la información.

3. Conocer la capacidad que tienen los estudiantes para investigar y resolver problemas en los sistemas y aplicaciones.

4. Conocer el dominio comunidades virtuales y redes sociales

\section{Marco Teórico}

La alfabetización tecnológica comprende el dominio de competencias y capacidades que permiten al estudiante tener un desempeño académico de calidad. De esta forma, esta temática se ha convertido en uno de los ejes centrales para los modelos educativos.

Por otro lado, de acuerdo con Ortega (2009), dicha alfabetización permite el proceso de democratización de los procesos de formación, además de afianzar la inclusión social, laboral y una considerable mejora en la calidad de vida.

Si bien es cierto que la evolución de las TIC supone una integración de distintas formas de aprendizaje, también ésta exige una construcción de entornos de interconexión de componentes informales y entornos institucionales.

$\mathrm{Al}$ respecto, Salinas (2015) menciona en su artículo "Hacia nuevas formas metodológicas en e-learning" que un entorno personal de aprendizaje permite al alumno tomar el control, además de decidir sus propios objetivos de aprendizaje, gestionar tanto el contenido como el proceso, comunicar con otros en el proceso de aprendizaje y todo aquello que contribuye al logro de los objetivos.
Ahora bien, es importante mencionar que la alfabetización tecnológica también puede considerarse como múltiple, ya que existen elementos coincidentes entre el mundo informático, los procesos de comunicación, los servicios de telecomunicaciones y el trabajo docente.

Así, Gutiérrez (2006) asevera que existen repercusiones sociológicas, económicas y políticas. La primera reside en el aumento en la cantidad de información, la velocidad de procesamiento y distribución. La segunda refiere a la distribución discriminada y la recepción individualizada e interactiva. Finalmente, la tercera comprende la aparición de nuevos lenguajes para comunicarse, las nuevas formas de conocer y pensar.

En lo que respecta al esquema multimodal tecnológico, es importante considerar la activación de las habilidades de pensamiento crítico y reflexivo, el fomento a la creatividad y el aprendizaje basado en proyectos escolares o trabajos integrados orientados a resolver problemáticas reales.

Para Gutiérrez (2006) los contenidos reflexivos se resumen a cuatro aspectos clave para la educación para los medios, tales como; la imagen no es la realidad, los medios reflejan intereses comerciales e ideológicos, los medios son importantes agentes de educación informal, las audiencias no sólo son consumidoras, sino productos de economía de mercado.

En lo que confiere al dominio del uso de las herramientas ofimáticas, es importante que el estudiante comprenda su complejidad, así como su gran funcionalidad, lo cual permite mejorar los procesos de planeación, operatividad, gestión y optimización de tiempo.

\section{Metodología}

La investigación tuvo un enfoque cuantitativo. Para ello se siguió la metodología recomendada por Hernández, Fernández \& Baptista, 2010, considerando que se ajustó a los criterios de que el problema sea observable y medible. 
El procedimiento utilizado, para la recolección de datos consistió en la aplicación del instrumento Cuestionario para el Estudio de la Competencia Digital del Alumnado de Educación Superior (CDAES) Gutiérrez, J.; Cabero, J.\& Estrada. L. (2017), como instrumento que permite evaluar la alfabetización tecnológica que muestran tener los estudiantes universitarios compartido previamente a través de la herramienta Google Drive. Asimismo, se trabajó en una Universidad de Cancún a través del Cuerpo Académico (CA) de Tecnologías de la Información y Comunicación, en el periodo mayo-agosto de 2019.

\section{Muestra}

La muestra objeto de estudio, fue seleccionada de entre los estudiantes universitarios, matriculados en algún grupo del programa educativo de TSU en TIC en una universidad de Cancún. Utilizando la técnica de muestreo no probabilística, denominada causal o accidental (Albert, 2006; Gil, Rodríguez, \& García, 2008), se configuró una muestra con un total de 21 estudiantes, de los cuales, el $95.23 \%$ pertenecían al género masculino (20 estudiantes) y el $4.76 \%$ restante al femenino (una alumna). La media de edad del alumnado correspondía a los 19 años, estando la mayoría dentro del intervalo que se encuentra entre los 19 y 21 años (el 77.27\%).

\section{Instrumento}

Para satisfacer los objetivos de nuestro estudio, se utilizó el Cuestionario para el Estudio de la Competencia Digital del Alumnado de Educación Superior (CDAES) Gutiérrez, J.; Cabero, J.\& Estrada. L. (2017), constituido por seis dimensiones que se desglosan en 22 indicadores, a partir de los cuales se concretan 44 ítems, y que para fines de nuestro estudio solamente utilizamos la dimensión uno Alfabetización tecnológica: Funcionamiento y conceptos de las TIC, la cual consiste en cuatro indicadores y 13 ítems, ver Tabla 1, los cuales permiten conocer qué saben y qué son capaces de hacer el alumnado del tercer cuatrimestre de TIC para aprender efectivamente y vivir productivamente en un mundo cada vez más digital.

\begin{tabular}{|c|c|}
\hline \multicolumn{2}{|c|}{$\begin{array}{l}\text { Dimensiones y número de indicadores en cada } \\
\text { dimensión del cuestionario CDAES }\end{array}$} \\
\hline $\begin{array}{l}\text { Dimensiones del } \\
\text { cuestionario }\end{array}$ & Indicadores \\
\hline \multirow{4}{*}{$\begin{array}{l}\text { Dimensión } 1 . \\
\text { Alfabetización } \\
\text { tecnológica } \\
\text { (Funcionamiento } \\
\text { conceptos } \\
\text { de las TICs) }\end{array}$} & $\begin{array}{l}\text { Entienden y usan sistemas } \\
\text { tecnológicos de Información } \\
\text { y Comunicación. }\end{array}$ \\
\hline & $\begin{array}{lrr}\text { Seleccionan } & \text { y } & \text { usan } \\
\text { aplicaciones efectiva } & \text { y } \\
\text { productivamente. } & \end{array}$ \\
\hline & $\begin{array}{l}\text { Investigan y resuelven } \\
\text { problemas en los sistemas y } \\
\text { las } \\
\text { aplicaciones. }\end{array}$ \\
\hline & $\begin{array}{l}\text { Transfieren el conocimiento } \\
\text { existente al aprendizaje de } \\
\text { nuevas tecnologías de } \\
\text { Información y Comunicación } \\
\text { (TIC). }\end{array}$ \\
\hline
\end{tabular}

Tabla 1

Fuente: Gutiérrez, J.; Cabero, J.\& Estrada. L. (2017)

Se decidió por una escala valorativa tipo Likert con 5 opciones de respuesta, debido a que le permite al alumnado posicionarse ante un abanico de posiciones (McMillan \& Schumacher 2010), a la vez que se omite un valor intermedio, con el fin de evitar su posible elección por el participante, motivado por su indecisión o indiferencia (Block \& Jones, 1968; Dubois \& Burns, 1975, cit. en Hernández, Espejo, González, \& Games, 2001; Gómez, 1991).

Así los sujetos pueden reflejar en una escala del 1 al 5 su grado de competencia tecnológica, donde el valor 1 hace referencia a que el estudiante se siente completamente ineficaz para realizar lo que se presenta, y 5 la dominación completa de la declaración.

Validez. El instrumento se validó por un panel de expertos compuesto por 17 profesores que impartían las asignaturas de "Tecnología Educativa" o/y "TIC aplicadas a la educación" en diferentes Universidades Españolas y Latinoamericanas, quienes revisaron $\mathrm{y}$ analizaron el contenido y la estructura del cuestionario, atendiendo especialmente a cuestiones de orden y con un interés especial puesto en evitar la acción de los posibles sesgos inherentes a los cuestionarios autoadministrados, por medio de la distribución de las preguntas dentro del cuestionario y de su redacción (Gutiérrez et al., 2017). 
Confiabilidad. Según el índice Alfa de Cronbach para el análisis de la coherencia interna, se obtuvo una fiabilidad de 0.902 . Por tanto, nos hallamos ante un valor más que suficiente para aceptar el uso de CDAES, de acuerdo a Hernández et al., (2010), quienes determinar que el valor mínimo para que un instrumento sea confiable es de .70, dicha consideración se encuentra apoyada por García Cadena, C. H. (2006).

\section{Estadísticas de fiabilidad.}

\begin{tabular}{|r|lr|}
\hline Alfa de Cronbach & N de elementos \\
\hline & .902 & \\
\hline
\end{tabular}

Tabla 2

Fuente: Elaboración propia, resultados con SPSS

\section{Procedimiento}

En el mismo momento los estudiantes responden al instrumento que se les proporciono mediante Google drive.

El tiempo de aplicación oscila entre 15 y 20 minutos, por lo que todos los datos se recogen en una única sesión de unos 30 minutos.

Los sujetos que responden son estudiantes de TIC que asisten a clase en aquel momento y que, de forma voluntaria acceden a colaborar en la investigación.

\section{Resultados}

Con respecto a la primera pregunta de investigación ¿Los estudiantes entienden y usan sistemas informáticos de comunicación e información?

De acuerdo con el instrumento CDAES los ítems que correspondieron para su análisis son del 1 al 3, por lo que se procedió a realizar la agrupación de estos ítems en una sola variable y mediante estadísticos descriptivos se puede observar que el porcentaje de estudiantes entienden y usan sistemas informáticos de comunicación e información "Bien" fue del 4.8 $\%$ y $95.5 \%$ como "Excelente". Ver tabla 3

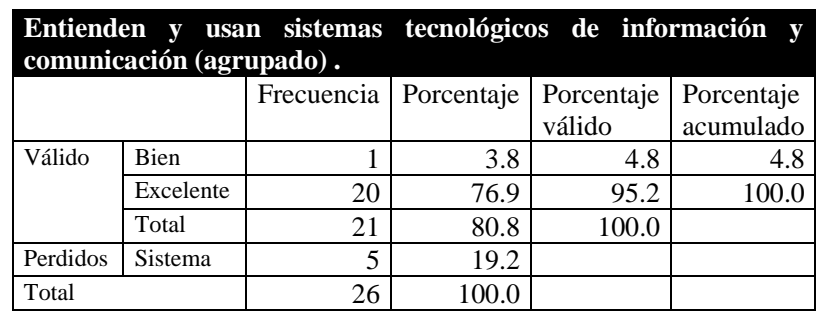

Tabla 3

Fuente: Elaboración propia, resultados con SPSS

En la segunda pregunta de investigación ¿Los estudiantes seleccionan y usan aplicaciones efectiva y productivamente? los resultados que se obtuvieron de los ítems 4 y del 6 al 11 agrupados en una sola variable y mediante estadísticos descriptivos se observó que los estudiantes seleccionan y usan aplicaciones efectiva y productivamente "Bien" en un $42.9 \%$ y $57.1 \%$ "Excelente". Ver tabla 4.

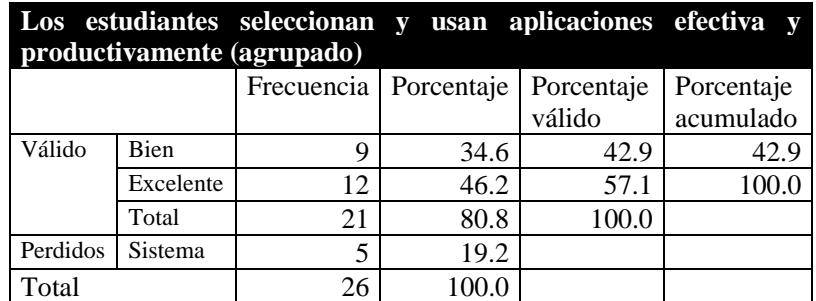

Tabla 4

Fuente: Elaboración propia, resultados con SPSS

Para la tercera pregunta ¿Los estudiantes investigan y resuelven problemas en los sistemas y las aplicaciones? Se consideró solo el ítem 5 que corresponde a las respuestas de los estudiantes que pueden configurar correo electrónico, configurar antivirus, desfragmentar el disco duro, en la Tabla 5 se muestra que el 9.5\% de los estudiantes investigan y resuelven problemas en los sistemas y las aplicaciones "Regular", el 47.6\% "Bien" y 42.9 "Excelente". Ver Gráfico 1.

\begin{tabular}{|c|c|c|c|c|c|}
\hline \multicolumn{6}{|c|}{$\begin{array}{l}\text { Investigo y resuelvo problemas en los } \\
\text { (configurar correo electrónico, } \\
\text { desfragmentar el disco duro) (agrupado). }\end{array}$} \\
\hline & & Frecuencia & Porcentaje & $\begin{array}{l}\text { Porcentaje } \\
\text { válido }\end{array}$ & $\begin{array}{l}\text { Porcentaje } \\
\text { acumulado }\end{array}$ \\
\hline \multirow[t]{4}{*}{ Válido } & Regular & 2 & 7.7 & 9.5 & 9.5 \\
\hline & Bien & 10 & 38.5 & 47.6 & 57.1 \\
\hline & Excelente & 9 & 34.6 & 42.9 & 100.0 \\
\hline & Total & 21 & 80.8 & 100.0 & \\
\hline Perdidos & Sistema & 5 & 19.2 & & \\
\hline \multicolumn{2}{|l|}{ Total } & 26 & 100.0 & & \\
\hline
\end{tabular}

Tabla 5

Fuente: Elaboración propia, resultados con SPSS. 




Gráfica 1 Porcentaje de estudiantes que investigan y resuelven problemas en los sistemas y aplicaciones Fuente: elaboración propia, elaboración con SPSS

Para la última pregunta de investigación ¿Los estudiantes transfieren el conocimiento existente al aprendizaje de nuevas tecnologías de Información y Comunicación? Se consideraron los Ítems 12 y 13 los cuales se agruparon en una sola variable y mediante el análisis descriptivo se observaron los siguientes resultados. Ver Tabla 6. El 9.5\% de los estudiantes transfieren el conocimiento, existente al aprendizaje de nuevas tecnologías de Información y Comunicación "Regular", el $66.7 \%$ "Bien" y el $23.8 \%$ "Excelente".

\begin{tabular}{|c|c|c|c|c|c|}
\hline \multicolumn{6}{|c|}{$\begin{array}{l}\text { Transferencia de conocimiento existente al } \\
\text { aprendizaie de nuevas tecnologías }\end{array}$} \\
\hline & & Frecuencia & Porcentaje & $\begin{array}{l}\text { Porcentaje } \\
\text { válido }\end{array}$ & $\begin{array}{l}\text { Porcentaje } \\
\text { acumulado }\end{array}$ \\
\hline \multirow[t]{4}{*}{$\begin{array}{l}\text { Válido } \\
\end{array}$} & Regular & 2 & 7.7 & 9.5 & 9.5 \\
\hline & Bien & 14 & 53.8 & 66.7 & 76.2 \\
\hline & Excelente & 5 & 19.2 & 23.8 & 100.0 \\
\hline & Total & 21 & 80.8 & 100.0 & \\
\hline Perdidos & Sistema & 5 & 19.2 & & \\
\hline \multicolumn{2}{|l|}{ Total } & 26 & 100.0 & & \\
\hline
\end{tabular}

Tabla 6

Fuente: elaboración propia, resultados con SPSS

\section{Conclusiones}

El análisis de los resultados que ofrece la presente investigación nos ha permitido dar respuesta a los objetivos planteados:

En cuanto al objetivo 1, han sido Identificadas las competencias que tienen los estudiantes para saber utilizar sistemas informáticos de comunicación e información de manera "Excelente" un 95.5 por ciento los estudiantes son capaces de utilizar distintos sistemas operativos en una computadora, de utilizar distintos dispositivos móviles y navegar por Internet en diferentes navegadores.
Con respecto al objetivo 2, se ha conocido que el 57.1 por ciento de los estudiantes tienen dominio en el uso de herramientas ofimáticas para el tratamiento de la información como distintas herramientas ofimáticas, distintas herramientas de tratamiento de imagen, de comunicación sincrónica, asincrónica, diseñan páginas web utilizando algún programa informático, usan software colaborativo, así como dominio de herramientas de la Web 2.0.

En cuanto al objetivo 3, se ha conocido que la capacidad que tienen los estudiantes para investigar y resolver problemas en los sistemas y aplicaciones es del 42.9 por ciento de manera "Excelente" y 47.6 por ciento como "Bien".

Y para el objetivo 4, se ha conocido el dominio comunidades virtuales y redes sociales es del 23.8 por ciento como "excelente" y 66.7 por ciento como "bien" ya que los estudiantes respondieron que usan eficazmente el sistema virtual su universidad y que se sienten competentes al utilizar la gestión virtual de su universidad.

Finalmente, podemos concluir que se identificado el nivel de alfabetización tecnológica en los estudiantes del tercer cuatrimestre en el programa educativo de Tecnologías de la Información y Comunicación en un 23.8\% el resultado fue "Bien" y el 76.2\% fue "Excelente". Ver Tabla 7.

\begin{tabular}{|c|c|c|c|c|c|}
\hline \multicolumn{6}{|c|}{ Alfabetización Tecnológica (agrupado) } \\
\hline & & Frecuencia & Porcentaje & $\begin{array}{l}\text { Porcentaje } \\
\text { válido }\end{array}$ & $\begin{array}{l}\text { Porcentaje } \\
\text { acumulado }\end{array}$ \\
\hline \multirow[t]{3}{*}{ Válido } & Bien & 5 & 19.2 & 23.8 & 23.8 \\
\hline & Excelente & 16 & 61.5 & 76.2 & 100.0 \\
\hline & Total & 21 & 80.8 & 100.0 & \\
\hline Perdidos & Sistema & 5 & 19.2 & & \\
\hline \multicolumn{2}{|l|}{ Total } & 26 & 100.0 & & \\
\hline
\end{tabular}

Tabla 7

Fuente: elaboración propia, resultados con SPSS

\section{Referencias}

García Cadena, C. H. (2006). La medición en las ciencias sociales y en psicología. Landeros Hernández, R. y González Ramírez, M. T. Estadística con SPSS y metodología de la investigación. México: Trillas. 
Gil, J., Rodríguez, G., \& García, E. (2008). Estadística básica aplicada a las Ciencias de la Educación. Sevilla: Kronos. Macmillan, J. \& Schumacher, S. (2010). Investigación educativa: Una introducción conceptual. Madrid: PearsonAddison Wesley.

Gisbert, M., Espuny, C., \& González, J. (2011). INCOTIC. Una herramienta para la @ utoevaluación diagnóstica de la Competencia digital en la universidad. Profesorado, Revista de currículum y formación del profesorado. Vol. $15, \mathrm{~N}^{\circ} 1$.

Gutiérrez, A. (2006) La alfabetización múltiple en la Sociedad de la Información. En el

Gutiérrez, J.; Cabero, J.\& Estrada. L. (2017): Diseño y validación de un instrumento de evaluación de la competencia digital del estudiante universitario, Revista Espacios, Vol. 8 No. 10. Universidad de Sevilla [Fecha de consulta: 26 de abril de 2019].https://idus.us.es/xmlui/bitstream/handle/ 11441/54725/Dise\%C3\%B1o_y_validacion_de _un_instrumento_de_evaluacion_de_la_compet encia_digital_del_estudiante.pdf?sequence $=1 \& \mathrm{i}$ sAllowed=y ISSN 07981015

Gutiérrez, M. (2002) Alfabetización tecnológica: competencias básicas para una nueva cultura, Dirección General de Universidades: Perspectivas de aplicación y desarrollo de las nuevas tecnologías de la Educación, Madrid, MECD, 23-30.

Hernández, A., Espejo, B., González, V., \& Gámez, J. (2001). Escalas de respuesta tipo likert. ¿es relevante la alternativa "indiferente"?. Metodología De Encuestas, 3(2), 135-150.

Hernández, S. R., Fernández, C. C. \& Baptista L. M. (2014). Metodología de la investigación (6a ed.). México: McGrawHill.

libro Claves de la Alfabetización Digital. Por Rafael Casado (Coord.). Editor(es): Ariel. Barcelona. 2006. ISBN 84-08-07120-3 , págs. 57-66. [Fecha de consulta: 26 de febrero del 2019].

file://C:/Users/G123/Downloads/Alfabetizacio nMltipleenSociedaddelaInformacin.pdf

Ortega Sánchez, I. (2009): La Alfabetización tecnológica”. En Ortega Sánchez, I. \& Ferrás Sexto, C. (Coord.) Alfabetización Tecnológica y el desarrollo regional [monográfico en línea].
Revista Electrónica Teoría de la Educación: Educación y Cultura en la Sociedad de la Información. Vol. 10, $\mathrm{n}^{\mathrm{o}}$ 2. Universidad de Salamanca [Fecha de consulta: 26 de febrero del 2019].

http://www.usal.es/ teoriaeducacion/rev_numer o_10_02/n10_02_ortega_sanchez.pdf ISSN: 1138-9737

Peña, I. (2006). Capacitación digital a la UOC: L'alfabetització tecnològica vs. La competència digital. En Jornades en xarxa sobre l'EEES. Universitat Oberta de Catalunya.

Salinas, I. (2015): "Hacia nuevas formas metodológicas en e-learning". [Fecha de consulta: 26 de febrero del 2019]. https://www.researchgate.net/publication/23224 2434_Hacia_nuevas_formas_metodologicas_en _E-Learning
PREZA-MEDINA, Sergio Roberto, HERNÁNDEZ-CHACÓN, Sandra, CEBOLLÓN-MEZA, Ángela y SAAVEDRARIVEROS, Érika. Alfabetización tecnológica en estudiantes de nivel superior. Revista Teoría Educativa. 2019 